\title{
Ephippial and subitaneous egg abortion: relevance for an obligate parthenogenetic Daphnia population
}

\author{
José M. CONDE-PORCUNA*, Francisco Javier VALDÉS, Susana ROMO ${ }^{1)}$ and Carmen PÉREZ-MARTÍNEZ \\ Institute of Water Research, University of Granada, Ramón y Cajal 4, 18071- Granada, Spain \\ ${ }^{1)}$ Area of Ecology, Faculty of Biology, Campus Burjasot, University of Valencia, 46100-Burjasot, Valencia. Spain \\ *e-mail corresponding author: jmconde@ugr.es
}

\begin{abstract}
We experimentally analysed the importance of egg abortion in the reproduction strategies of Daphnia pulicaria (Forbes) in Lake Rio Seco, a high mountain lake. Adult animals were exposed to different treatments under continuous or dynamic conditions (temperature and photoperiod) to test whether the simulation of field conditions, with changing temperature and photoperiod, induces differences in their reproductive traits in comparison to continuous conditions, with the aim of extrapolating results to the lake. Continuous conditions were the photoperiod and temperature values recorded in the lake at the time when ephippial reproduction is induced. Dynamic conditions were the changing values in the field from the continuous conditions until autumn. The number of healthy ephippial eggs per ephippium was also recorded by using sediment traps in the lake. Our results show that subitaneous and ephippial egg abortion has a major influence on an obligate parthenogenetic Daphnia population in both the laboratory and field. The total number of healthy ephippial eggs per ephippium (mean $\pm S E ; 0.52 \pm 0.12$ ) and the proportion of released empty ephippia obtained in the sediment traps $(0.73 \pm 0.05)$ were similar to those experimentally obtained under dynamic conditions (healthy eggs per ephippium: $0.29 \pm 0.16$; proportion of released empty ephippia: $0.79 \pm 0.11$ ), suggesting that ephippial egg abortion and empty ephippia production are frequent in nature. This is the first study to describe Daphnia ephippial egg abortion and compare subitaneous and ephippial egg abortion between experimental and field conditions. This approach is recommended for future research into Daphnia or cladoceran reproduction.
\end{abstract}

Key words: high-mountain lake, reproduction strategies, ephippial eggs, egg abortion, Daphnia pulicaria

\section{INTRODUCTION}

The number of subitaneous eggs in the Daphnia brood chamber is commonly used for estimating population growth rate in field zooplankton studies (Seitz 1979; Keen \& Nassar 1981; Mooij et al. 2003). This number is also a reasonably good predictor of food availability in field studies. However, eggs may be aborted when they fail to develop, producing an overestimation of released egg numbers and therefore an overestimation of the population growth rate and food availability in aquatic systems (Razlutskij 2000).

Little attention has been paid to the egg abortion rate in Daphnia population dynamics and reproduction studies, despite frequent observation of the degeneration or abortion of parthenogenetic eggs in natural habitats (Brooks 1946; Hall 1964; Threlkeld 1985). Threlkeld (1979) demonstrated that egg abortion can be considerable at certain periods (e.g., during cyanobacteria blooms). Subitaneous egg abortion can be more readily quantified in the laboratory than in the field. The limited number of studies on this issue have highlighted the inaccuracies produced by the failure to include egg abortion in Daphnia population dynamics research (Boersma \& Vijverberg 1995; Vijverberg et al. 1996; Weers \& Gulati 1997; Urabe \& Sterner 2001).
The abortion of ephippial eggs would also be significant in Daphnia population studies. They may be produced by sexual Daphnia populations or by obligate parthenogenetic Daphnia populations. In high-mountain lakes, with a harsh climate and short growing season, individuals may ensure persistence by rapidly investing in dormant eggs to ensure persistence (Decaestecker et al. 2009). This strategy is more easily accomplished by obligate parthenogenetic species, since no males are needed to fertilize the eggs, whereas sexual Daphnia must pay the cost of producing males (Maynard Smith 1978; Innes et al. 2000; Decaestecker et al. 2009). The absence of a mating constraint for obligate parthenogens may favour an increased allocation to asexual diapausing eggs earlier in the season in comparison to cyclical parthenogens, which require mating with males to produce sexual diapausing eggs (Innes et al. 2000). However, no studies on ephippial egg abortion have been performed with either sexual or obligate parthenogenetic populations of Daphnia.

Ephippial reproductive rates calculated by ephippial counts alone may be overestimated if ephippial egg abortion or empty ephippia produced by Daphnia are not taken into account. Moreover, when the long-term persistence of Daphnia depends on ephippial egg production and hatching, this overestimation may have a especial impact on our understanding of the ecological 
and evolutionary dynamics of the population (Marcus et al. 1994; Weider et al. 1997; Hairston 1999).

Food quantity and quality have been proposed as the main factors controlling subitaneous egg abortion (Richman 1958; Boersma \& Vijverberg 1995; Urabe \& Sterner 2001), while Stearns (1987) and Lively \& Johnson (1994) described parthenogenetic egg abortion as a strategy to obtain the maximum viable progeny through the abortion of defective embryos.

With this background, we experimentally analysed the egg abortion of $D$. pulicaria, an obligate parthenogenetic population, from Lake Río Seco (southern Spain). We tested egg abortion under continuous and dynamic conditions. The continuous conditions were the values of photoperiod and temperature recorded at the time that environmental conditions induce ephippial reproduction (middle August). Dynamic conditions were the changing values from the continuous conditions until autumn. The objective was to determine the importance of egg abortion in Daphnia population and whether the simulation of field conditions by changing temperature and photoperiod induces different results in comparison to continuous conditions. We measured not only the number of ephippia produced but also the ephippial egg abortion rate, which we considered crucial to estimate the role of ephippial reproduction in the population dynamics of $D$. pulicaria. In a preliminary experiment, sediment traps were deployed during the ice-free period to quantify the number of healthy ephippial eggs per ephippium and the proportion of empty ephippia produced by Daphnia in the lake.

\subsection{Study site}

Río Seco is a small $\left(1920 \mathrm{~m}^{2}\right)$, oligotrophic and shallow $\left(\mathrm{Z}_{\max }=2.90 \mathrm{~m}\right)$ high mountain lake $(3020 \mathrm{~m}$ a.s.l.) of glacial origin located in the Sierra Nevada mountains (southern Spain). Lake Río Seco is a harsh but a relatively predictable habitat, which favours asexuality in Daphnia (Decaestecker et al. 2009). The lake is ice covered from October-November until JuneJuly. Temperature ranges from approximately 4 to $16^{\circ} \mathrm{C}$ during the ice-free period. The lake is without fish and presents total Secchi disk visibility over the ice-free period.

The plankton community of the lake is extremely simple. There are around 10 nanoplanktonic species of algae, and the dominant species are Chromulina nevadensis (P.M. Sánchez), Ochromonas sp., Dyctiosphaerium chlorelloides (Nauman) and zoospores of the epizoan chlorophyte Korshikoviella gracilipes (Lambert). The zooplankton community is dominated by the calanoid Mixodiaptomus laciniatus (Lilljeborg) and the cladoceran D. pulicaria.

D. pulicaria population in Río Seco is an obligate parthenogenetic population. Hatchlings emerge from the ephippial eggs immediately after the thaw. D. pulicaria usually reproduces by subitaneous eggs until mid-Au- gust, when there is a shift to ephippial egg production (Barea-Arco et al. 2001; Pérez-Martínez et al. 2007).

\section{METHODS}

\subsection{D. pulicaria reproduction experiment}

A laboratory experiment was performed using the physical conditions (temperature and photoperiod) as independent factors. The food for the experimental animals was the chlorophyte Scenedesmus acutus (Meyen), grown in a batch culture with B5282 medium (SigmaAldrich Inc., St Louis, MO, USA), middle aeration and a day-length of 14 hours. This edible algae (biovolume from 31 to $184 \mu^{3}$ ) is not present in the lake, but other edible chlorophytes are very common (biovolume from 4 to $170 \mu \mathrm{m}^{3}$; Pérez-Martínez, unpublished data), and $S$. acutus can be considered food of sufficiently good quality (DeMott \& Müller-Navarra 1997; Young et al. 1997; Zhang et al. 2009). The food concentration usually recorded in the Río Seco Lake $\left(0.1 \mathrm{mg} \mathrm{C} \mathrm{L}^{-1}\right)$ was selected (Barea-Arco et al. 2001, Pérez-Martínez, unpublished data).

The physical conditions were designed to compare continuous conditions with dynamic conditions. The continuous conditions simulated the temperature and photoperiod conditions in the lake at the time of experimental animal collection $\left(14.5{ }^{\circ} \mathrm{C}\right.$ and $13 \mathrm{~h} 40$ min light day ${ }^{-1}$ ). The dynamic conditions simulated the changes in field temperature and day length, starting with the continuous condition values, which then reduced by $0.12^{\circ} \mathrm{C} \mathrm{day}^{-1}$ and $1.1 \mathrm{~min}$ of light day ${ }^{-1}$ until the end of the experiment.

D. pulicaria individuals were collected with vertical plankton net tows from Lake Río Seco on 14 August 2006, around the time that ephippial egg production starts to be observed in the lake (Pérez-Martínez et al. 2007). After $24 \mathrm{~h}$ of acclimation in the laboratory, adult females without subitaneous or ephippial eggs were selected (length $>1500 \mu \mathrm{m}$ ). Using a longer acclimation time to constant temperature and photoperiod by culturing several Daphnia generations in the laboratory would have interfered with the purpose of the experiment, since changing conditions of temperature and photoperiod served to simulate field conditions. Each individual was placed in a $100 \mathrm{~mL}$ glass tube containing $75 \mathrm{~mL}$ of filtered and autoclaved lake water. A total of 72 selected animals were randomly assigned to one of the two treatments under study (36 individuals per treatment).

The experiment lasted for 23 days, checking the animals daily for survival, egg production and moults. When moulting was detected, the animal was examined under inverted microscope (40× magnification) to record the presence and type of eggs before it was returned to its original tube. Half of the water volume in each tube was replaced weekly with fresh filtered and autoclaved lake water. Food (S. acutus) was added on alternate days, and experimental tubes were replaced 
with the same frequency to avoid accumulation of algae at their ends.

For each individual, we recorded the eggs produced ( ${ }^{\circ}$ in egg chamber or ephippium on the day after moult) and the eggs released $\left(\mathrm{n}^{\circ}\right.$ developed and released as newborns or $\mathrm{n}^{\circ}$ diapausing eggs in released ephippium). The number of aborted eggs was calculated by deducting $\mathrm{n}^{\circ}$ eggs released from $\mathrm{n}^{\circ}$ eggs produced. The following study variables were therefore obtained: ephippia produced, empty ephippia produced, subitaneous eggs produced, ephippial eggs produced, empty ephippia released, subitaneous eggs released, ephippial eggs released, subitaneous eggs aborted, and ephippial eggs aborted.

\subsection{Sediment traps}

The lake area was divided into five areas of around the same size, placing two sediment traps at a random point within each area from 27 July to 3 November. The sediment traps, plastic cylinders of $31 \mathrm{~cm}$ height and $2.25 \mathrm{~cm}$ radius (de Vicente et al. 2008), were placed at the bottom of the lake and sampled weekly. The trap content was filtered through a $30 \mu \mathrm{m}$ mesh and immediately preserved with $70 \%$ ethanol. The Daphnia ephippia in the traps were counted in the laboratory, The number of healthy ephippial eggs in each ephippium was obtained by opening it with a pair of dissecting needles and counting all eggs that were not evidently degenerated (Cáceres 1998).

\subsection{Data analysis}

The STATISTICA programme (Statsoft) was used for the data analyses. Survival was analysed by using a survival test for censored data and multiple samples (Kirk 1997), treating surviving animals at the end of the assay as censored data. Differences in survival function between pairs of treatments were tested by using the nonparametric log-rank test for homogeneity.

Only surviving animals were considered for the analysis of the effects of physical conditions on Daphnia subitaneous and ephippial egg production. Because the distribution of data was abnormal, a non-parametric test was used (Mann-Whitney U test).

\section{RESULTS}

\subsection{Survival and reproduction}

The mean size of selected animals was $1950.81 \pm$ $39.14 \mu \mathrm{m}$ (mean \pm SE). A total of 22 animals died over the 23 experimental days, leaving 50 survivors at the end of the assay; survival curves showed no differences among treatments (Fig. 1).

Reproduction was observed in $92 \%$ of the survivors, with ephippial production in $72 \%$ and subitaneous production in 54\%. Table 1 shows the total number of eggs produced and released with each treatment.

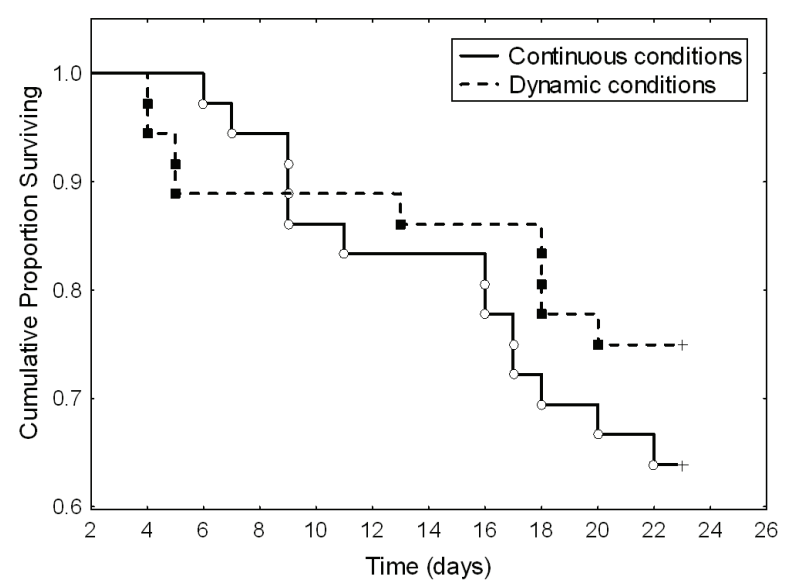

Fig. 1. Number of surviving animals in continuous and dynamic conditions (o Complete data + Censored data). Logrank statistics $=0.96, p>0.05$.

Tab. 1. Total number of produced and released eggs (subitaneous or ephippial) in each treatment: DC, Dynamic conditions; CC, Continuous conditions.

\begin{tabular}{cccccc}
\hline & \multicolumn{2}{c}{$\mathrm{N}^{\circ}$ subitaneous eggs } & & \multicolumn{2}{c}{$\mathrm{N}^{\circ}$ ephippial eggs } \\
\cline { 2 - 3 } \cline { 5 - 6 } & produced & released & & produced & released \\
\hline $\mathrm{CC}$ & 171 & 92 & & 37 & 14 \\
$\mathrm{DC}$ & 33 & 24 & & 18 & 5 \\
\hline
\end{tabular}

The number of ephippial eggs produced per individual and the number of subitaneous eggs per individual did not significantly differ between the dynamic and continuous conditions (Fig. 2A). However, the number of subitaneous and ephippial eggs released per individual was significantly higher under continuous versus dynamic conditions (Fig. 2B).

Calculating the egg abortion rates as the ratio of aborted eggs to eggs per individual, we observed that both the subitaneous and ephippial egg abortion rates were significantly higher under dynamic versus continuous conditions (Fig. 3).

\subsection{Sediment traps}

The mean number ( \pm SE) of healthy ephippial eggs per ephippium from July to November was $0.52 \pm 0.12$, and the total number of released ephippia was 120 . In the period from August to September (experiment period), the mean number of healthy ephippial eggs per ephippium was $0.50 \pm 0.23$. In the experiment, the number of ephippial eggs per ephippium did not significantly differ between treatments $\left(\mathrm{Z}_{\text {adjusted }}=1.874, p\right.$ $>0.05$ ) and was similar to the number observed in the field (continuous conditions: $0.68 \pm 0.18$, dynamic conditions: $0.29 \pm 0.16$ ).

Under dynamic conditions, the proportion of empty ephippia released was significantly higher than under continuous conditions (Fig. 4A) but similar to observations in the field (Figs 4A and 4B), where an elevated proportion was recorded in the sediment traps throughout the study period (Fig. 4B). 

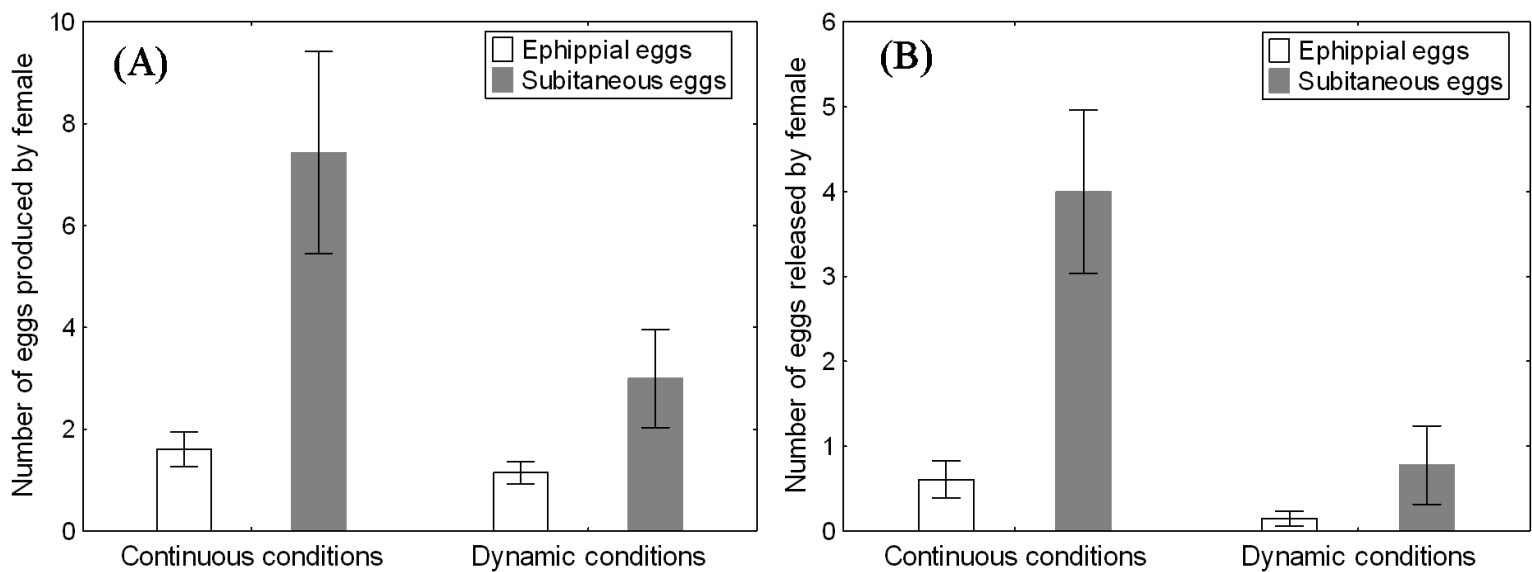

Fig. 2. Total number of eggs produced $(\mathbf{A})$ or released $(\mathbf{B})$ per individual (mean $\pm \mathrm{SE}$ ) in each treatment. A: Mann-Whitney U Test did not show any significant effect of physical conditions on the number of eggs produced (subitaneous eggs: $\mathrm{Z}_{\text {adjusted }}=1.722, \mathrm{n}=50, p$ $>0.05$; ephippial eggs: $Z_{\text {adjusted }}=0.807, \mathrm{n}=50, p>0.05$ ). B: Mann-Whitney $U$ Test shows a significant effect of physical conditions on the number of eggs released (subitaneous eggs: $\mathrm{Z}_{\text {adjusted }}=3.377, \mathrm{n}=50, p<0.001$; Ephippial eggs: $\mathrm{Z}_{\text {adjusted }}=2.044, \mathrm{n}=50, p$ $<0.05)$.

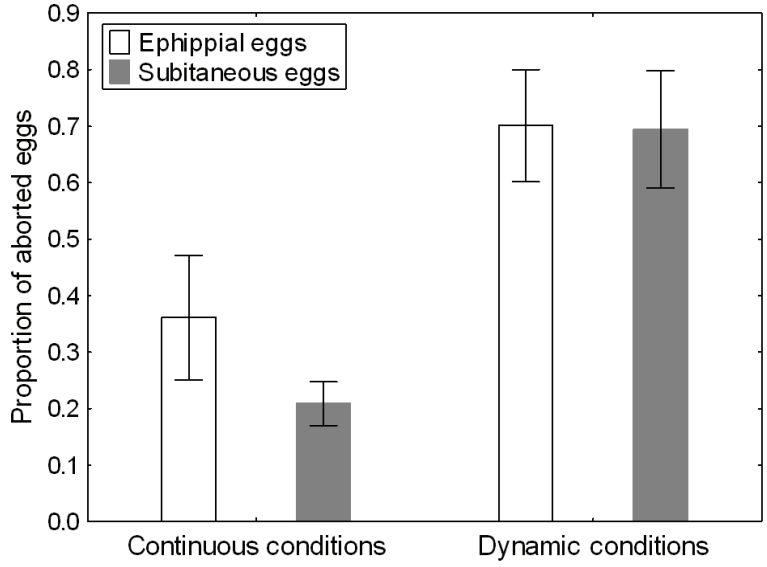

Fig. 3. Ephippial and subitaneous egg abortion rates (eggs aborted / eggs produced) per individual (mean $\pm \mathrm{SE}$ ) in each treatment. Subitaneous abortion rates (Mann-Whitney $\mathrm{U}$ Test): $\mathrm{Z}_{\text {adjusted }}=3.391, \mathrm{n}=27, p<0.001$. Ephippial abortion rates (Mann-Whitney $\mathrm{U}$ Test): $\mathrm{Z}_{\text {adjusted }}=2.239, \mathrm{n}=32, p<0.05$.
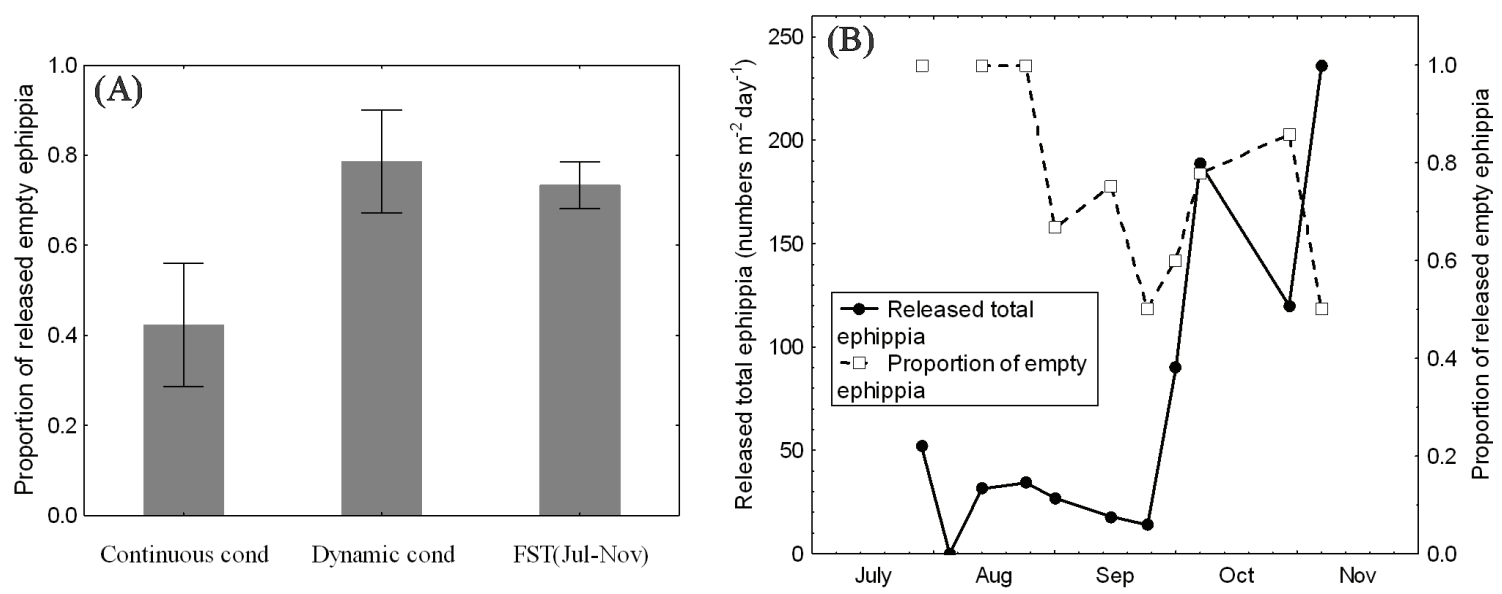

Fig. 4. Proportion of empty ephippia released by Daphnia. (A) Proportion (mean \pm SE) obtained in the experiment and in the field sediment traps (FST). Continuous vs dynamic conditions (Mann-Whitney U Test): $\mathrm{Z}_{\text {adjusted }}=1.972, \mathrm{n}=27, p<0.05$. (B) Changes over time in the number of ephippia and in the proportion of empty ephippia released in Lake Río Seco. 
The empty ephippia observed in the sediment traps may have included ephippia that were produced already empty and those losing their eggs by abortion. In the experiment, most of the empty ephippia released by Daphnia resulted from the abortion of resting eggs (Fig. 5).

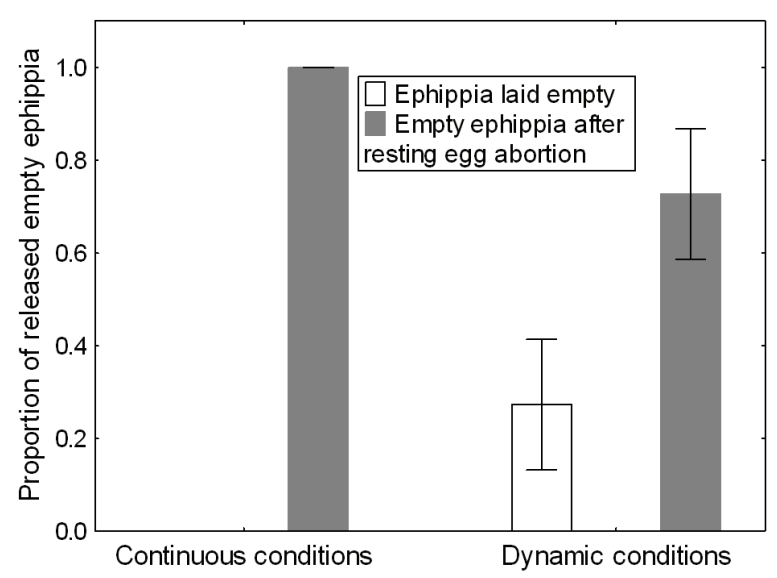

Fig. 5. Relative contributions of ephippial egg abortion and empty ephippia production to the empty ephippia released.

\section{DISCUSSION}

This is the first report to demonstrate the relevance of subitaneous and ephippial egg abortion in a natural system. The degeneration or abortion of parthenogenetic eggs has been observed in Daphnia populations in natural habitats (Brooks 1946; Redfield 1981; Threlkeld 1985), with some authors reporting that $>50 \%$ of eggs in Daphnia populations were non-viable (Boersma \& Vijverberg 1995). Nevertheless, ephippial egg abortion has not previously been investigated. The ephippial reproduction of Daphnia has been studied by considering the number of ephippia produced (Abrusán et al. 2007; Koch et al. 2009). However, our field and experimental results show that the number of healthy eggs per ephippium can be much lower than the two eggs assumed in previous studies, implying that ephippial reproduction has been overestimated, compromising our understanding of Daphnia reproduction and long-term persistence in natural systems.

No difference in survival was observed between the continuous and dynamic conditions. The mortality during the experiment was within the range previously reported for this species from the same lake (BareaArco et al. 2001). The production of ephippia represented an energy cost for $D$. pulicaria individuals, suggesting a decrease in the allocation of energy to survival (Threlkeld 1987, Barea-Arco et al. 2001).

Ephippial and subitaneous egg production was observed under both continuous and dynamic conditions, but ephippial and subitaneous egg abortion rates were higher under dynamic conditions. One possible explanation is that eggs produced under specific physical conditions may be aborted if these conditions change, as occurs in nature. Numerous studies have analysed the effect of food and/or physical conditions on Daphnia egg production, but none have taken account of their effect on egg abortion and, therefore, on viable offspring production. Moreover, although the temperature and photoperiod in the continuous conditions could be expected to be more favourable for Daphnia than the gradual decline of temperature and light in the dynamic conditions, our experimental results indicated that experimental studies on ephippial production under continuous conditions overestimate the production of viable offspring.

The causes of egg abortion or degeneration have not been determined, although some authors have proposed that subitaneous egg abortion rates are influenced by the quantity and/or quality of food (Brooks 1946; Richman 1958; Boersma \& Vijverberg 1995). However, Urabe \& Sterner (2001) observed subitaneous abortion under optimal food conditions. Other authors found that toxicants produced partial or complete abortion of clutches of subitaneous eggs in Daphnia (i.e., Baird et al. 1991). However, the present study is the first to demonstrate that physical conditions (photoperiod and temperature) are related to subitaneous egg abortion and ephippial egg abortion in this species.

If egg abortion is due to the deficiency of a substance essential for healthy egg development, it has been questioned (Urabe \& Sterner 2001) why Daphnia do not decrease the size of the brood to favour the development of all eggs. Urabe \& Sterner (2001) suggested that Daphnia may use egg abortion to balance the elemental or biochemical composition of their body tissues. Stearns (1987) suggested that the overproduction of zygotes in brooding organisms is favoured in general because it increases the average viability of progeny through the selective abortion of inferior embryos. Zygote overproduction greatly increases the probability that a rare parthenogenetic mutant will spread to fixation in comparison to non-brooding parthenogens (Johnson et al. 1995). Another possibility is that inferior offspring may be selectively aborted and replaced (Lively \& Johnson 1994), although the replacement of embryos implies a cost. A strategic model of selective abortion in parthenogenetic organisms indicated that the range of values that favours the spread of parthenogenesis increases with the number of times that aborted eggs are replaced (Lively \& Johnson 1994). These possible strategies may be more likely in species with obligate parthenogenesis, such as Daphnia pulicaria in our study, which may have a higher proportion of mutants in comparison to cyclic parthenogenetic species. Hence, when ephippial reproduction occurs, Daphnia individuals may attempt to produce the maximum number of ephippial eggs. This response could produce defective eggs that are later aborted.

Our data on the number of ephippial eggs per ephippium and on the proportion of empty ephippia obtained in the lake, using sediment traps, are similar to those 
experimentally obtained under dynamic conditions (Fig. 4). We observed in both cases a low number of healthy ephippial eggs per ephippium and a high number of empty ephippia, which suggests high abortion rates in the lake and /or low production of eggs per ephippium. Hence, the dynamic conditions of our experiment represent well the field situation. In our experiment, we also observed that resting egg abortion was the main factor causing the release of empty ephippia by Daphnia, since only a small fraction of released empty ephippia were already produced empty.

Fox (2007) observed a high proportion of empty ephippia in recent sediments. She suggested that the loss of eggs from ephippia is likely due to higher rates of hatching in recent years rather than the deterioration of eggs over time, although she considered it impossible to rule out differences in fertilization or early egg death without a closer examination of ephippial contents. Vaníčková et al. (2010) pointed out that the occurrence of empty ephippia does not necessarily indicate previous hatching events. In sexual Daphnia populations, a certain proportion of ephippia are always laid empty when not fertilized (Zaffagnini 1987). Vaníčková et al. (2010) showed that this proportion varies substantially among localities and species: It was estimated to be at least $10 \%$ in a North American lake inhabited by two Daphnia species (Cáceres 1998), and around 25\% of ephippia were produced empty by $D$. galeata in a Czech reservoir (Vaníčková et al., unpublished data). In our study, more than $70 \%$ of ephippia were released empty in the lake (field sediment traps) and under dynamic experimental conditions. Keller et al. (2007) reported annual empty ephippia proportions of $32 \%$ and $50 \%$ in two subsequent years for a hybridizing assemblage of $D$. longispina and D. galeata.

These authors suggest that empty ephippia are a common phenomenon in sexual reproduction in Daphnia. However, we describe here that empty ephippia are also relevant in an obligate parthenogenetic Daphnia species, and that the presence of empty ephippia is due not only to empty laying but also to resting egg abortion. As ephippia are more frequently produced by obligate asexual than sexual genotypes (Heier \& Dudycha 2009), there may also be differences in resting egg abortion between sexual and asexual Daphnia populations. Innes et al. (2000) commented that obligate parthenogens may show a higher investment in diapausing eggs compared with cyclical parthenogens, although they observed that obligate parthenogens did not appear to differ from cyclical parthenogens in ephippia formation, despite the lack of a mating constraint. However, these authors did not examine differences in resting eggs, and the cost of producing males by cyclical parthenogens may be compensated for by an increase in the number of viable resting eggs per ephippium. In summary, we encourage further research on resting egg abortion in sexual Daphnia populations.

\section{CONCLUSIONS}

Elucidation of the mechanisms of Daphnia diapause requires in-depth knowledge of the natural system in question. Estimation of ephippial egg abortion may be a powerful method for understanding the reproduction strategy of natural populations. In the present study, the proportion of ephippial and subitaneous aborted eggs was more than $20 \%$ under continuous conditions and around $70 \%$ under dynamic conditions. Field results for empty ephippia and ephippial eggs were similar to those obtained under dynamic conditions, indicating that previous studies may have overestimated the reproduction rates (subitaneous and ephippial) of Daphnia, regardless of food conditions. This is the first scientific study to measure the ephippial egg abortion rate. This approach can be recommended for future research into zooplankton reproduction. Further studies are warranted to evaluate the mechanisms underlying the abortion of Daphnia eggs.

\section{ACKNOWLEDGMENTS}

Financial support was obtained from CICYT Project CGL2004-03031/BOS and MMA Projects 129B/2003 and $87 / 2007$. The authors wish to thank all colleagues who have helped to collaborate in the experiment and maintenance of the cultures. They are grateful to Dr. Eloisa Ramos-Rodríguez for advice on algal culture and to Ricardo Corro and Belén Herrador for their assistance in the collection of the animals and the maintenance of their cultures. We also thank two anonymous reviewers for making valuable suggestions to earlier drafts of this study. The authors are grateful to Richard Davies for linguistic improvements.

\section{REFERENCES}

Abrusán, G., P. Fink, \& W. Lampert. 2007. Biochemical limitation of resting egg production in Daphnia. Limnol. Oceanogr., 52: 1724-1728.

Baird, D.J., I. Barber, MC. Bradley, A.M.V.M. Soares \& P. Calow. 1991 A comparative study of genotype sensitivity to acute toxic stress using clones of Daphnia magna Straus. Ecotoxicol. Environ. Safety, 21: 257-265.

Barea-Arco, J., C. Pérez-Martínez \& R. Morales-Baquero. 2001. Evidence of a mutualistic relationship between an algal epibiont and its host, Daphnia pulicaria. Limnol. Oceanogr., 46: 871-881.

Boersma, M. \& J. Vijverberg. 1995. The significance of nonviable eggs for Daphnia population dynamics. Limnol. Oceanogr., 40: 1215-1224.

Brooks, J.L. 1946. Cyclomorphosis in Daphnia. I. An analysis of D. retrocurva and D. galeata. Ecol. Monograph, 16: 409-447.

Cáceres, C.E. 1998. Interspecific variation in the abundance, production, and emergence of Daphnia diapausing eggs. Ecology, 79: 1699-1710.

Decaestecker, E., L. De Meester \& J. Mergeay. 2009. Cyclical parthenogenesis in Daphnia: sexual versus asexual reproduction. In: I Schoen, K Martens \& PJ Van Dijk (Eds), Lost sex: the evolutionary biology of parthenogenesis. Springer Press Chapter 15: 295-316. 
DeMott, W.R. \& D.C. Müller-Navarra. 1997. The importance of highly unsaturated fatty acids in zooplankton nutrition: evidence from experiments with Daphnia, a cyanobacterium and lipid emulsions. Freshwat. Biol., 38: 649-664

de Vicente, I., F. Rueda, L. Cruz-Pizarro \& R. MoralesBaquero. 2008. Implication of settling process on phosphorus dynamic in three contrasting reservoirs. Fundam. Appl. Limnol.,170: 263-272

Fox, J.A. 2007. Hatching timing of Daphnia mendotae diapausing eggs of different ages. Arch. Hydrobiol., 168: 19-26.

Hall, D.J. 1964. Experimental approach to dynamics of natural population of Daphnia galeata mendotae. Ecology, 45: 94-112.

Hairston, N.G. Jr. 1999. Rapid evolution revealed by dormant eggs. Nature, 401: 446.

Heier, C.R. \& J.L. Dudycha. 2009. Ecological speciation in a cyclic parthenogen: Sexual capability of experimental hybrids between Daphnia pulex and Daphnia pulicaria. Limnol. Oceanogr., 54: 492-502.

Innes, D.J., C.J. Fox, G.L. Winsor. 2000. Avoiding the cost of males in obligately asexual Daphnia pulex (Leydig). Proc. R. Soc. Lond. [Biol.], 267: 991-997.

Johnson, S.G., C.M. Lively \& S. J. Schrag. 1995. Evolution and ecological correlates of uniparental reproduction in freshwater snails. Experientia, 51: 498-509.

Keen, R. \& R. Nassar. 1981. Confidence intervals for birth and death rates estimated with the egg ratio technique for natural populations of zooplankton. Limnol. Oceanogr., 26: 131-142.

Keller, B., J. Wolinska, C. Tellenbach \& P. Spaak. 2007. Reproductive isolation keeps hybridizing Daphnia species distinct. Limnol. Oceanogr., 52: 984-991.

Kirk K.L. 1997. Life-history responses to variable environments: Starvation and reproduction in planktonic rotifers. Ecology, 78: 434-441.

Koch, U., E. von Elert \& D. Straile. 2009. Food quality triggers the reproductive mode in the cyclical parthenogen Daphnia (Cladocera). Oecologia, 159: 317-324.

Lively, C.M. \& S.G. Johnson. 1994. Brooding and the evolution of parthenogenesis strategy models and evidence from aquatic invertebrates. Proc. R. Soc. Lond. [Biol.], 256: 89-95.

Marcus, N.H., R. Lutz, W. Burnett \& P. Cable. 1994. Age, viability, and vertical-distribution of zooplankton resting eggs from an anoxic basin - Evidence of an egg bank. Limnol. Oceanogr., 39: 154-158.

Maynard Smith, J.M. 1978. The evolution of sex. Cambridge University Press. 236 pp.

Mooij, W.M., S. Hulsmann, J. Vijverberg, A. Veen \& E. Lammens. 2003. Modeling Daphnia population dynamics and demography under natural conditions. Hydrobiologia, 491: 19-34.

Pérez-Martínez C., J. Barea-Arco, J.M. Conde-Porcuna \& R. Morales-Baquero. 2007. Reproduction strategies of Daph- nia pulicaria population in a high mountain lake of Southern Spain. Hydrobiologia, 594: 75-82.

Razlutskij, V.I. 2000. Estimating cladoceran birth rate: use of the egg age distribution to estimate mortality of ovigerous females and eggs. Hydrobiologia, 428: 135-144.

Redfield, G.W. 1981. Nutrition and the degeneration of eggs in a limnetic daphnid. Int. Ver. Theor. Angew. Limnol. Verh., 21: 1550-1554.

Richman, S. 1958. The transformation of energy by Daphnia pulex. Ecol. Monograph, 28: 274-291.

Seitz, A. 1979. Calculation of birth rates and death rates in fluctuating populations with continuous recruitment. Oecologia, 41: 343-360.

Stearns, S.C. 1987. The selection-arena hypothesis. Experientia Suppl., 55: 337-349.

Threlkeld, S.T. 1979. Estimating cladoceran birth rates. Importance of egg mortality and the egg age distribution. Limnol. Oceanogr., 24: 601-612.

Threlkeld, S.T. 1985. Egg degeneration and mortality in cladoceran populations. Int. Ver. Theor. Angew. Limnol. Verh., 22: 3083-3087.

Threlkeld, S.T. 1987. Daphnia life history strategies and resource allocation patterns. In: Peters, R. H. \& R. de Bernardi (Eds), Daphnia. Mem. Ist. ital. Idrobiol., 45: 353-366.

Urabe J. \& R.W. Sterner. 2001. Contrasting effects of different types of resource depletion on life-history traits in Daphnia. Funct. Ecol., 15: 165-174.

Vaníčková I., J. Seda \& A. Pterusek. 2010. The stabilizing effect of resting egg banks of the Daphnia longispina species complex for longitudinal taxon heterogeneity in long and narrow reservoirs. Hydrobiologia, 643: 85-95.

Vijverberg, J., D.F. Kalf \& M. Boersma. 1996. Decrease in Daphnia egg viability at elevated pH. Limnol. Oceanogr., 41: 789-794.

Weider L.J., W. Lampert, M. Wessels, J.K. Colbourn \& P. Limburg. 1997. Long-term genetic shifts in a microcrustacean egg bank associated with anthropogenic changes in the Lake Constance ecosystem. Proc. R. Soc. Lond. [Biol.], 264: 1613-1618.

Weers, P.M.M., \& R.D. Gulati. 1997. Growth and reproduction of Daphnia galeata in response to changes in fatty acids, phosphorus, and nitrogen in Chlamydomonas reinhardtii. Limnol. Oceanogr., 42: 1584-1589.

Young, S., M. Palm, J.P. Grover \& D. McKee. 1997. How Daphnia cope with algae selected for inedibility in longrunning Microcosms. J. Plankton Res., 19: 391-397.

Zaffagnini, F., 1987. Reproduction in Daphnia. In: Peters, R. H. \& R. de Bernardi (Eds), Daphnia. Mem. Ist. ital. Idrobiol., 45: 245-284.

Zhang, X., T.W. Warming, H.Y. Hu \& K.S. Christoffersen. 2009. Life history responses of Daphnia magna feeding on toxic Microcystis aeruginosa alone and mixed with a mixotrophic Poterioochromonas species. 2009. Water Res., 43: 5053-5062. 